\title{
Applications of Scanning Transmission X-ray Microscopy (STXM) to Industrially Relevant Polymeric Materials
}

\author{
D.A. Winesett* and P.A. Stevens** \\ *ExxonMobil Chemical Company, Baytown Polymers Center, 5200 Bayway Drive, Baytown, Texas, 77520- \\ 2101. \\ **ExxonMobil Research and Engineering, Corporate Strategic Research, Clinton Township, Route 22E, \\ Annandale, New Jersey 08801.
}

Optimizing materials of commercial interest to the polymer industry often requires a detailed understanding of the structure-property relationship at a sub-micron level. The necessary balance of properties and cost usually dictates that these materials be multi-component systems of several polymers along with various fillers and additives. Therefore, the ability to study the complex microstructure in these materials at high chemical and spatial resolution is essential. One characterization technique available for such characterization is Scanning Transmission X-ray Microscopy (STXM). The primary attributes of STXM for applications to polymers are the ability to chemically identify and map materials at spatial resolution well below $100 \mathrm{~nm}$ (30 nm in some cases). STXM utilizes Near Edge X-ray Absorption Fine Structure (NEXAFS) in transmission to provide quantitative chemical identification and eliminates the need for staining, selective dissolution, or special sample preparation necessary for contrast.

As an example of how STXM is beneficial to our research efforts, data is presented from a non-commercial model blend system made with $36 \mathrm{wt} . \%$ butadiene rubber (BR), $36 \mathrm{wt} . \%$ brominated poly(isobutylene-co-4methylstyrene) (BIMS), and $28 \mathrm{wt} . \%$ silica filler. C1s NEXAFS reference spectra from each of the constituent components are shown in Figure 1 [1]. Peaks at $285.2 \mathrm{eV}$ and $287.9 \mathrm{eV}$ indicate the presence of $\mathrm{C}=\mathrm{C}$ and $\mathrm{C}-\mathrm{H}$ bonding, respectively, and can provide excellent contrast for imaging BR and BIMS. Sections of the blended material were prepared by cryo-microtomy, placed on $\mathrm{Si}_{3} \mathrm{~N}_{4}$ window, and imaged with the SUNY@Stony Brook STXM. Figure 2 shows a series of STXM micrographs (converted to total x-ray absorbance, or optical density) acquired at the energies indicated on the spectra. Because the BIMS copolymer only contains a few weight percent of 4-methylstyrene, most of the absorbance mapped in Figure $2 \mathrm{~b}$ is due to BR and silica. Furthermore, Figure $2 \mathrm{a}$ maps only silica and Figure $2 \mathrm{c}$ predominately maps the BIMS. To further identify and quantify the polymer morphology and location of filler, we solve for each component's mass thickness by fitting a Beer law absorbance law on a pixel-by-pixel basis to the series of images and NEXAFS cross sections from the reference spectra. Figure 3 shows the compositional map of silica (Figure 3a), BR (Figure 3b) and BIMS (Figure 3c). Figure 4 shows the same maps from a different area of the same sample where the silica is more finely dispersed. One of the questions to be answered for this system is in which phase the dispersed silica ultimately resides. Careful inspection of the maps show that most of the finely dispersed silica is contained in BIMS phases while the largest accumulations of silica are mixed with BR (seen clearly in Figure $3 \mathrm{~b}$ ). Using both of these data sets, we calculate that $75 \%$ of the dispersed silica is contained in BIMS phases, results consistent with AFM experiments using elastic modulus contrast to identify the phases and location of the silica. However, the largest agglomerations of silica (such as the one shown in Figure 3) cannot be measured with AFM without damaging the tip. The compositional maps indicate that the largest silica phases are contained in nearly pure BR phases, a result otherwise unavailable [2].

[1] BR spectrum used with permission from H. Ade research Group; see O Dhez, S. Urquhart, and H. Ade, Journal of Electron Spectroscopy 128(1) 85 (2003).

[2] The authors would like to thank J. Ball for cryo-microtomy and A.J. Dias for samples and suggestions. 

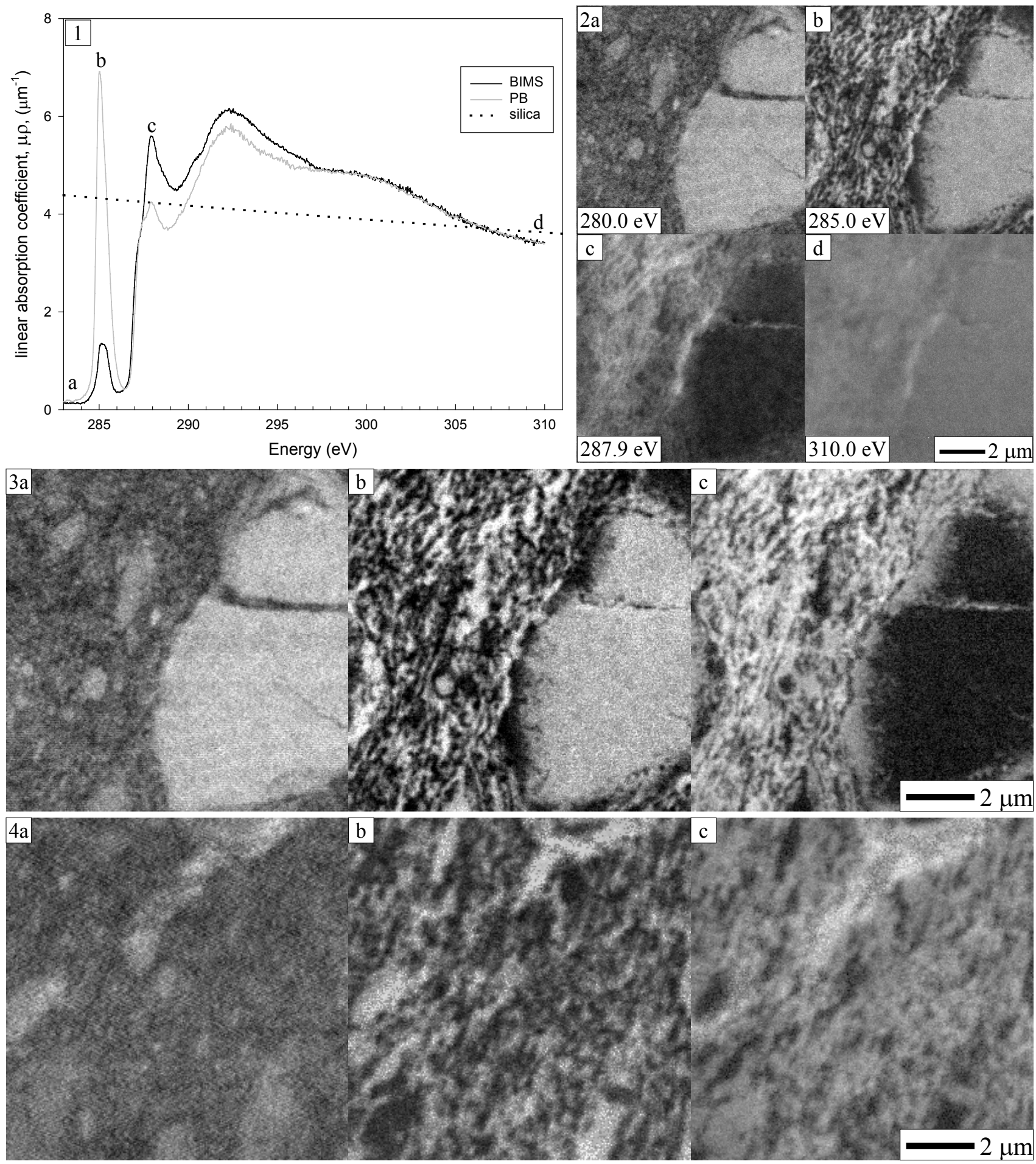

Figure 1 NEXAFS spectra from constituent components.

Figure 2 Total x-ray absorbance maps of BR/BIMS/silica blend acquired at energies indicated in Figure 1.

Figure 3 Compositional map of a) silica, b) BR, and c) BIMS.

Figure 4 Compositional map of a) silica, b) BR, and c) BIMS from a second location in the sample.

Copyright 2003 Exxon Mobil Corporation. All rights reserved. 